\section{Relações entre Ensino e Pesquisa em Epidemiologia (com comentários sobre a estratégia do mestrado em consórcio da UFPel)}

\section{Eduardo Faerstein}

Instituto de Medicina Social da UERJ
A Comissão de Epidemiologia da Abrasco oportunamente incluiu, na programação do seminário metodológico dedicado aos estudos seccionais (SP, abril 2007), mesa-redonda sobre o ensino da disciplina em suas inter-relações com as atividades de pesquisa epidemiológica. $\mathrm{O}$ compromisso com a melhoria do ensino da Epidemiologia no país está nas origens da Abrasco e inscrito no nome da nossa associação.

Cabe-me comentar a bem-sucedida estratégia do mestrado em consórcio do Programa de Pós-Graduação em Epidemiologia da Universidade Federal de Pelotas (UFPel), apresentada por Aluísio J.D. Barros no seminário ${ }^{1}$. Faço isso à luz de algumas considerações preliminares, e também da experiência a respeito do grupo de pesquisa que coordena o Estudo PróSaúde ${ }^{2}$.

Nosso pano de fundo sobre o tema tem duas premissas: é desejável uma forte articulação entre pesquisa e ensino, e a articulação entre essas duas vertentes da prática epidemiológica pode ser benéfica para ambas, especialmente em países como o nosso. Mas o que se observa?

- Houve enorme crescimento da literatura epidemiológica nas duas últimas décadas. Livros, por exemplo: hoje em dia, quem quer aprender ou ensinar epidemiologia tem uma profusão de livros à disposição. Há relativamente pouco tempo não era assim. Até o início da década de 1980, essas fontes podiam ser contadas nos dedos de uma das mãos ${ }^{3-5}$. Realizei consulta (em 30/07/2007) na base de dados da mais conhecida livraria virtual (http://www.amazon.com), buscando títulos que incluíssem a palavra "epidemiology" ou "epidemiologia”, chegando a 1.945 títulos em inglês e 74 em português ou espanhol quase igual ao que cheguei com "astrofísica” (2.254 e 17 títulos, respectivamente), e mais do que com "antropologia cultural” (1.687 e 76). Apesar dessa riqueza editorial, pouco tem sido debati-

Endereço para correspondência: Eduardo Faerstein. Rua Sacopã, 191 - apto 20. Rio de Janeiro, RJ Cep 22471-180. E-mail: eduardof@ims.uerj.br 
do ou mesmo relatado sobre o ensino da Epidemiologia, que implicasse em alguma sistematização crítica da experiência acumulada. Isso pode ser também verificado em buscas nas bases de dados Medline, Scielo ou Scholar Google: existem poucas referências a respeito, a maioria versando sobre o ensino de Epidemiologia na graduação médica ${ }^{6-8}$.

- No Brasil, na década de 1990 começamos a ter nossos congressos de epidemiologia da Abrasco, e a elaboração dos Planos Diretores para o Desenvolvimento da Epidemiologia no Brasil ${ }^{9,10}$ a cada qüinqüênio. Segundo Guimarães et al ${ }^{11}$, ao final dessa década passada 176 grupos e 813 pesquisadores (2/3 com menos de 10 anos de doutoramento) haviam registrado, no Diretório dos Grupos de Pesquisa no Brasil (DGP-CNPq, versão 4.0, 2000), 320 linhas de pesquisa epidemiológica. Mas, também em nosso país, a vitalidade da disciplina não se traduziu ainda em esforços mais consistentes de consolidação das experiências havidas no ensino de Epidemiologia. Há referências esparsas, incluídas por exemplo na proposição do currículo de curso de saúde pública ${ }^{12}$, e em considerações sobre a capacitação necessária à avaliação de programas ${ }^{13}$. Apenas Barata ${ }^{14}$ conduziu há 10 anos mapeamento mais abrangente das tendências a respeito. Entretanto, sobre o tema específico desse debate, isto é, como aperfeiçoar relações entre a pesquisa epidemiológica e o ensino da disciplina, aparentemente nada se publicou em periódicos indexados após os relatos das experiências dos inquéritos domiciliares conduzidos em Ribeirão Preto (nos anos 1970) ${ }^{15}$ e Campinas (anos 1980) ${ }^{16}$. O mais recente Plano Diretor para o Desenvolvimento da Epidemiologia no Brasil (IV Plano, para o período 20052009) não aborda o tema ${ }^{10}$.

- No DGP-CNPq, 813 pesquisadores em Epidemiologia registraram 940 estudantes, nos diversos níveis de forma- ção, como vinculados a suas atividades de pesquisa ${ }^{14}$. Cerca de metade desses era de estudantes de graduação, e metade de pós-graduandos (Gráfico): em média, 0,52 aluno de graduação por pesquisador, 0,37 de mestrado e 0,21 de doutorado. Essas médias sugerem baixa densidade de inserção de estudantes nas atividades de pesquisa epidemiológica no país. Guimarães et al. ${ }^{11}$ sugeriram que a relação entre doutorandos e pesquisadores doutores indicaria o "dinamismo" de uma área de conhecimento (por indicar a intensidade de reprodução da força de trabalho); por sua vez, a relação entre o conjunto de doutorandos e todos os estudantes vinculados aos pesquisadores mediria indiretamente seu grau de "consolidação”. O Quadro 1 (seleção de alguns dados apresentados pelos autores) sugere que, desse ponto de vista, a Epidemiologia brasileira estaria em fase dinâmica (intermediária entre o conjunto da Saúde Coletiva e as Ciências Exatas), mas ainda apresentaria baixa consolidação científica (de modo similar ao conjunto da Saúde Coletiva, mas inferior ao das Ciências Exatas).

\section{A estratégia do mestrado em consórcio da UFPel}

É no contexto acima delineado que reside a importância do detalhado relato apresentado por Barros ${ }^{1}$ sobre as premissas, a implementação prática e os resultados alcançados com a estratégia do mestrado em consórcio da UFPel. Como de hábito, os epidemiologistas brasileiros estarão atentos também a esse relato dos colegas de Pelotas. As evidências apresentadas são muito convincentes, no sentido de que a estratégia de consórcio de pesquisa possibilitou o cumprimento a contento das duas idéias centrais do programa ("formar epidemiologistas que tivessem experiência de campo e soubessem não só analisar dados, mas também planejar e conduzir estudos de campo; e di- 


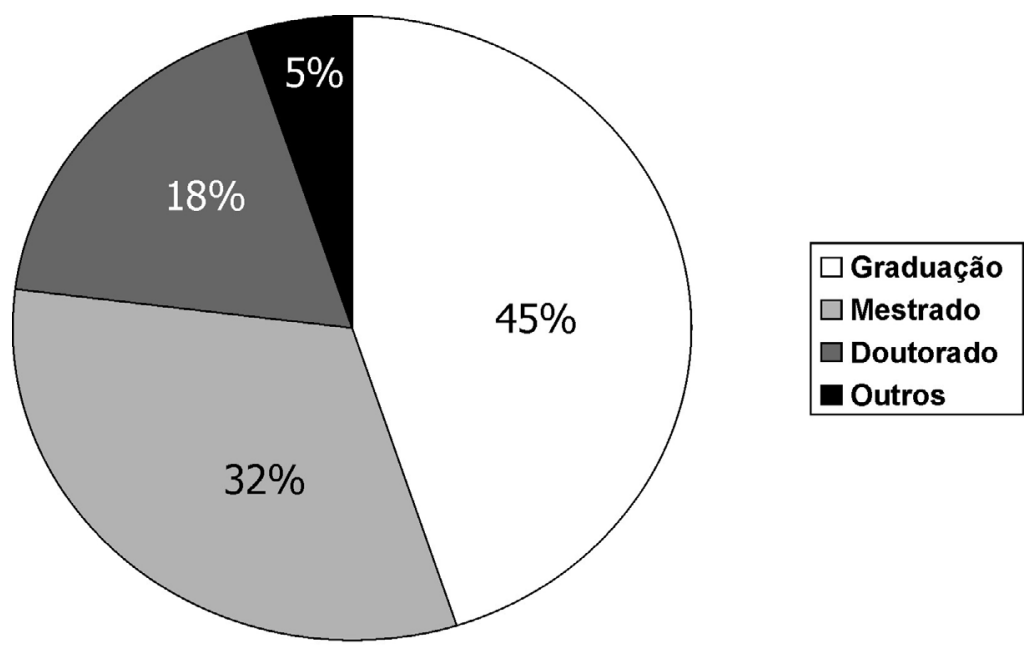

Fonte: Guimarães 2001 , DGP-CNPq versão 4.0

Gráfico - Estudantes ( $n=940)$ orientados por pesquisadores $(n=813)$ em Epidemiologia segundo nível de treinamento. Brasil, 2000.

Graph - Students $(n=940)$ supervised by researchers $(n=813)$ in Epidemiology according to level of training. Brazil, 2000.

Quadro 1 - Relações entre alunos e pesquisadores doutores em grupos de pesquisa: Epidemiologia, Saúde Coletiva, Ciências Exatas. Brasil, 2000

Chart 1 - Relations between students and doctorate researchers in research groups: Epidemiology, Collective Health, Exact Sciences. Brazil, 2000

\begin{tabular}{lcc}
\hline Áreas & $\begin{array}{c}\text { doutorandos/pesquisadores doutores } \\
\text { ("dinamismo") }\end{array}$ & $\begin{array}{c}\text { doutorandos/total alunos } \\
\text { ("consolidação") }\end{array}$ \\
\hline Epidemiologia & 0,40 & 0,18 \\
Saúde Coletiva & 0,32 & 0,17 \\
Ciências Exatas & 0,47 & 0,30 \\
\hline
\end{tabular}

vulgar de forma efetiva os resultados de pesquisas"). Com uso de indicadores objetivos, após o inicio dessa estratégia foi possível mensurar objetivamente melhorias, tanto na proporção e tempo de titulação, como na intensidade e possivelmente na qualidade das publicações resultantes. Explicações alternativas para essas tendências favoráveis não parecem plausíveis: por exemplo, um aumento concomitante da qualidade do corpo docente esse já era de alta qualidade no período anterior; o mesmo quanto ao corpo discente - não há indicações de mudanças no tipo de clientela e nos critérios de seleção.

A seguir, após descrever brevemente o
Estudo Pró-Saúde e as atividades de ensino de Epidemiologia nele desenvolvidas, comentaremos algumas possíveis vantagens e desvantagens comparativas das duas estratégias.

\section{Estudo Pró-Saúde}

Em 1999 iniciamos no Estado do Rio de Janeiro um estudo prospectivo de funcionários públicos de campi universitários, com interesse temático principal em torno dos determinantes sociais da saúde e de comportamentos de saúde. Em 2001 foi completada a coleta de dados de base da coorte; dessas 2 fases (1999-2001), partici- 
param 3.253 funcionários com idade entre 22-69 anos (média 42 anos), com distribuição equilibrada quanto a sexo (56\% mulheres) e raça ( $48 \%$ pretos e pardos), e com razoável heterogeneidade socioeconômica (escolaridade: até $1^{\circ}$ grau, $24 \%$; $2^{\circ}$ grau, $36 \%, 3^{\circ}$ grau, $40 \%$; renda domiciliar per capita: < 3 salários mínimos (SM), 34\%; 3-6 SM, 35\%, > 6 SM, 31\%. No momento (julho de 2007), estamos concluindo nova fase de coleta de dados, já alcançando $90 \%$ dos participantes das fases anteriores. Foi utilizado questionário autopreenchível, e aferidos peso, estatura, circunferência da cintura e pressão arterial. Estimamos que, no período 1998-2007, o projeto (coordenado, além do autor, pelos Profs. Dóra Chor, Claudia S. Lopes e Guilherme L. Werneck) implicou em custos diretos de cerca de 750 mil reais. Outros detalhes podem ser encontrados em Faerstein et al. ${ }^{2}$.

\section{Atividades de ensino de Epidemiologia no Estudo Pró- Saúde}

No período considerado, tiveram inserção no projeto 89 alunos: 45 bolsistas de Iniciação Científica (graduação de Ciências Sociais, Serviço Social, Estatística, Informática, Enfermagem, Medicina); 20 alunos de mestrado - 100\% titulação (15/ 15); 21 alunos de doutorado - 88\% titulação (14/16), e 3 pós-doutorandos.

Os estudantes desempenham, sob supervisão docente, tarefas inerentes a cada etapa do estudo: desenho e pré-testes dos questionários; aplicação de questionários e aferições; atividades de controle de qualidade; processamento, gerência e análise de dados, entre outras. O trabalho de mestrandos e doutorandos teve papel fundamental em aspectos importantes do planejamento e da execução do estudo, como, por exemplo, a adaptação transcultural de escalas de apoio social e de estresse no trabalho; recrutamento, seleção, treinamento, certificação e supervisão de aferidores de pressão arterial e antropometria, entre outros. Essa inserção propiciou tanto maior densidade na formação acadêmica desses alunos, como o barateamento dos custos de pessoal do projeto.

Nos Seminários de Pesquisa Pró-Saúde (SPPS), realizados quinzenalmente (uma disciplina eletiva de 30 horas a cada semestre: SPPS I-VIII), há apresentação e discussão das análises em andamento nas dissertações, teses e artigos; debate de temas teóricos (por exemplo, determinantes sociais da saúde) e metodológicos (por exemplo, em semestres recentes: interação, imputação múltipla, análise de dados ordinais, modelos multinível), e avaliação das tarefas desempenhadas em campo.

\section{Possíveis vantagens e desvantagens comparativas das duas estratégias}

Essa comparação (Quadro 2) não leva em conta que, provavelmente, doutorandos do Programa da UFPel (experiência não relatada no seminário) tem vinculação com pesquisa com mais elementos em comum com a nossa estratégia.

No consórcio da UFPel, os alunos são expostos a todo o espectro de atividades de um projeto de pesquisa: formulação de pergunta, produção e processamento de dados; além disso, têm maior liberdade de escolha do tema de pesquisa. Sua experiência de grupo de pesquisa, entretanto, parece limitar-se aos orientadores e demais mestrandos e, nesse sentido, sua atividade de pesquisa pode contribuir pouco para o desenvolvimento do(s) grupo(s) de pesquisa da instituição, visto que a cada nova turma reinicia-se o processo. Por fim, dedicam-se apenas à análise de dados seccionais, o que não pode efetivamente ser considerado uma limitação para a formação no nível de mestrado.

No Estudo Pró-Saúde, os alunos têm menor liberdade de escolher seu tema de pesquisa. Isso é equacionado em conversas preliminares nos Departamentos de Epidemiologia do Instituto de Medicina 
Quadro 2 - Aspectos comparativos da experiência pesquisa-ensino em Epidemiologia: Mestrado em Consórcio UFPel e Estudo Pró-Saúde

Chart $\mathbf{2}$ - Comparative aspects of the research-teaching experience in Epidemiology:UFPel and Pro Health Study Consortium

\begin{tabular}{|c|c|c|}
\hline Aspecto da experiência & Consórcio Pelotas & Estudo Pró-Saúde \\
\hline Liberdade de escolha do tema & Maior & Menor \\
\hline Dados analisados & Seccionais & Seccionais ou longitudinais \\
\hline $\begin{array}{l}\text { Experiência no grupo } \\
\text { de pesquisa }\end{array}$ & $\begin{array}{l}\text { Com orientadores e } \\
\text { demais mestrandos }\end{array}$ & $\begin{array}{c}\text { Com orientadores e } \\
\text { bolsistas IC, mestrandos, } \\
\text { doutorandos, pós-doutorandos }\end{array}$ \\
\hline $\begin{array}{l}\text { Contribuição para crescimento } \\
\text { do grupo de pesquisa }\end{array}$ & $\begin{array}{l}\text { Processo reiniciado a } \\
\text { cada nova turma }\end{array}$ & Processo contínuo \\
\hline $\begin{array}{l}\text { Amplitude da experiência } \\
\text { de pesquisa }\end{array}$ & $\begin{array}{l}\text { Abrange todas as etapas } \\
\text { da pesquisa }\end{array}$ & $\begin{array}{l}\text { Pode limitar-se a etapa } \\
\text { em andamento }\end{array}$ \\
\hline
\end{tabular}

Social-UERJ e da Escola Nacional de Saúde Pública-FIOCRUZ, quando alunos são alocados a orientadores e grupos de pesquisa levando em conta seus interesses. Dependendo da fase em que esse estudo longitudinal se encontre, a participação discente pode se limitar à etapa em andamento - por exemplo, não coincidindo com o trabalho de campo. Por outro lado, em todos os níveis de formação os alunos interagem com orientadores e bolsistas de Iniciação Científica, mestrandos, doutorandos e pós-doutorandos. Na medida em que participam de um processo contínuo, todos podem contribuir para a formulação de perguntas de pesquisa sugeridas por análises anteriores, podendo em princípio analisar dados seccionais ou longitudinais.

Concluindo, as escolhas das melhores estratégias para integrar atividades de pesquisa e ensino em Epidemiologia dependerão sempre de considerações, por vezes complexas, em torno da natureza dos projetos de pesquisa existentes ou possíveis em cada instituição, assim como da situação dos programas de ensino de graduação e pós-graduação. Tendo em vista a heterogeneidade sabidamente existente a respeito no país, cabe intensificar o registro detalhado das experiências em curso, como foi aqui feito pelos colegas da UFPel. Como em muitos outros países, é fundamental que as pressões por produtividade em pesquisa e publicações a que somos crescentemente submetidos no ambiente acadêmico não continuem a implicar riscos para o aperfeiçoamento do ensino. Como vertente nobre da praxis acadêmica, o ensino deve estar alinhado à descoberta, à integração e à aplicação do conhecimento epidemiológico ${ }^{17}$. Quem sabe, os editais das agências de financiamento poderiam incluir, como critério adicional de avaliação de projetos de pesquisa, aspectos como participação discente, e mesmo produção de material didático relacionado ao projeto?

\section{Referências}

1. Barros, AJD. O Mestrado em Consórcio do Programa de Pós-Graduação em Epidemiologia da UFPel: uma experiência inovadora. Rev Brasil Epidemiol 2008; 11 (Supl. 1): 133-44.
2. Faerstein E, Chor D, Lopes CS, Werneck GL. Estudo PróSaúde: características gerais e aspectos metodológicos. Rev Brasil Epidemiol 2005; 8: 454-66. 
3. Morris JN. Uses of Epidemiology. Edinburgh: Livingstone; 1957

4. McMahon B, Pugh TF. Epidemiology: Principles and Methods. Boston: Little Brow and Co.; 1970

5. Armijo-Rojas R. Epidemiologia. Buenos Aires: Inter Medica; 1974.

6. Terris M. The teaching of epidemiology to medical students. Arch Environ Health 1966; 12: 801-13.

7. Ernster VL. On the teaching of epidemiology to medical students. Am J Epidemiol 1979; 109: 617-8.

8. Grufferman S, Kimm SY, Maile MC.Teaching epidemiology in medical schools: a workable model. Am J Epidemiol 1984; 120: 203-9.

9. Abrasco. III Plano diretor para o desenvolvimento da epidemiologia no Brasil 2000-2004. Rev Bras Epidemiol 2000; 3: 70-93.

10. Abrasco. IV Plano Diretor 2005-2009. Rev Bras Epidemiol 2005; 8(Supl. 1): 18-27.

11. Guimarães R, Lourenço R, Cosac S. A pesquisa em epidemiologia no Brasil. Rev Saúde Pública 2001; 35(4): $321-40$.
12. Souza JMP, Mercadante OA, Arantes GR, Ferreira AS, Vasconcelos TP. Curso de Saúde Pública em um semestre: algumas considerações. Rev Saude Publica 2006; 40: 772-7.

13. Hartz ZMA, Camacho LAB. Formação em epidemiologia para avaliação de programas. Cad Saúde Pública 1996; 12(Supl. 2): 13-20.

14. Barata RB. Tendências do ensino de Epidemiologia no Brasil. Rev Panam Salud Publica 1997; 2: 334-41.

15. Carvalheiro JR, Sanches O. Amostragem domiciliar continua em estudos epidemiológicos e no ensino. Rev Saude Pública 1979; 13: 195-202.

16. Barros MB, Carvalheiro JR. Entrevistas domiciliárias e o ensino e pesquisa em epidemiologia. Rev Saude Publica 1984; 18: 411-7.

17. Hofmeyer A, Newton M, Scott C. Valuing the scholarship of integration and the scholarship of application in the academy for health sciences scholars: recommended methods. Health Research Policy and Systems 2007, 5:5 doi:10.1186/1478-4505-5-5. 